\title{
Validity of ultrasound muscle thickness measurements for predicting leg skeletal muscle mass in healthy Japanese middle-aged and older individuals
}

\author{
Yohei Takai ${ }^{*}$, Megumi Ohta ${ }^{2}$, Ryota Akagi ${ }^{3}$, Emika Kato ${ }^{4}$, Taku Wakahara ${ }^{5}$, Yasuo Kawakami ${ }^{6}$,
}

Tetsuo Fukunaga ${ }^{1}$ and Hiroaki Kanehisa ${ }^{1}$

\begin{abstract}
Background: The skeletal muscle mass of the lower limb plays a role in its mobility during daily life. From the perspective of physical resources, leg muscle mass dominantly decreases after the end of the fifth decade. Therefore, an accurate estimate of the muscle mass is important for the middle-aged and older population. The present study aimed to clarify the validity of ultrasound muscle thickness (MT) measurements for predicting leg skeletal muscle mass (SM) in the healthy Japanese middle-aged and older population.

Findings: MTs at four sites of the lower limb and the bone-free lean tissue mass (LTM) of the right leg were determined using brightness-mode ultrasonography and dual-energy X-ray absorptiometry (DXA), respectively, in 44 women and 33 men, 52- to 78-years old. LTM was used as a representative variable of leg skeletal muscle mass. In the model-development group (30 women and 22 men), regression analysis produced an equation with $\mathrm{R}^{2}$ and standard error of the estimate (SEE) of 0.958 and $0.3 \mathrm{~kg}$, respectively: $L T M(\mathrm{~kg})=0.01464 \times\left(M_{\text {SUM }} \times \mathrm{L}\right)\left(\mathrm{cm}^{2}\right)-2.767$, where $M T_{\text {sum }}$ is the sum of the product of MTs at four sites, and $L$ is length of segment where MT is determined. The estimated LTM $(7.0 \pm 1.7 \mathrm{~kg})$ did not significantly differ from the measured LTM $(7.0 \pm 1.7 \mathrm{~kg})$, without a significant systematic error on a Bland-Altman plot. The application of this equation for the cross-validation group (14 women and 11 men) did not yield a significant difference between the measured (7.2 $\pm 1.6 \mathrm{~kg}$ ) or estimated $(7.2 \pm 1.6 \mathrm{~kg})$ LTM and systematic error.
\end{abstract}

Conclusion: The developed prediction equation may be useful for estimating the lean tissue mass of the lower extremity for the healthy Japanese middle-aged and older population.

Keywords: Dual-energy X-ray absorptiometry, Multiple regression analysis, Bland-Altman plot, Cross-validation

\section{Background}

Age-related loss in leg skeletal muscle mass (SM) accelerates after the end of the fifth decade [1]. This links to the greater influence of aging in the strength capability of the lower compared to the upper limb muscles [2-4], and consequently, to the augmentation of muscular effort in performing the activities of daily living in older individuals [5]. Furthermore, the strength capability of the lower

\footnotetext{
* Correspondence: y-takai@nifs-k.ac.jp

${ }^{1}$ National Institute of Fitness and Sports in Kanoya, 1 Shiromizu, Kanoya, Kagoshima 891-2393, Japan

Full list of author information is available at the end of the article
}

limb muscles is associated with cognitive function [6], and the declines in these abilities lead to disabilities in older people [7]. Therefore, accurate measurement of leg SM in the middle-aged and older population is critical to assess their mobility in daily life.

At present, computerized tomography (CT), magnetic resonance imaging (MRI) and dual-energy X-ray absorptiometry (DXA) are widely used as reference methods for evaluating SM in vivo. However, these methods are impractical in field studies examining large populations. Brightness-mode (B-mode) ultrasonography has the same advantage as CT or MRI in visualizing fat and

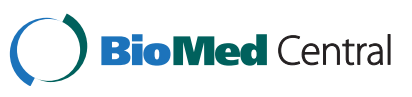


muscle tissues without compression and has been successfully used to evaluate muscle thickness (MT) in an older population [8]. In addition, the B-mode MT measurements are easily applicable in clinical and field surveys with no hazardous effects [9], and the measured values can be significant predictors of limb muscle volume $[10,11]$. These facts and findings support the applicability of B-mode MT measurements for assessing limb SM in a field survey examining a large sample. To the best of our knowledge, however, the only previous report to have examined the validity of the MT-based prediction equation in a sample including older people is that of Miyatani et al. [10]. In addition, their study examined only men and tested only knee extensor muscles. Thus, the applicability of MT measurement for predicting leg SM in older people remains questionable.

The current study aimed to clarify the applicability of ultrasound-based MT measurement for estimating leg bone-free lean tissue mass (LTM) in healthy Japanese middle-aged and older people. To this end, we determined leg LTM by DXA and assumed it to be a valid variable for assessing the leg SM on the basis of previous reports [12-14]. Miyatani et al. [11] indicated that ultrasound-based MT measurement was a good predictor of limb muscle volume when combined with limb length. Thus, we hypothesized that the product of MT with limb length should be a strong contributor for predicting the measured LTM.

\section{Methods}

\section{Subjects}

Forty-four women and 33 men, 52- to 78-years old, participated in this study. None of the subjects was or had been an athlete. Moreover, none was using walking sticks or other walking aids and all were functionally independent in daily life. In addition, no participants were on extreme diets or were using any major medications, such as chemotherapy, cardiac, respiratory, or antipsychotic drugs. A holdout sample validation method was used to develop and test the validity of the new equations. The sample in the current study $(\mathrm{n}=77)$ was split randomly into a model-development group (30 women and 22 men) and a cross-validation group (14 women and 11 men) (Table 1). According to a previous study [15], the ratio of the model-development group to the cross-validation group in the number of the subjects was two to one. This study was approved by the Ethics Committee on Human Research of Waseda University and was consistent with their requirements for human experimentation. The subjects were fully informed of the purpose and risks of the experiment and gave their written informed consent.

\section{Muscle thickness measurements}

MTs at four sites (thigh anterior and posterior, lower leg anterior and posterior) on the right leg were determined using a real time B-mode ultrasound apparatus (SSD-900, Aloka Co., Tokyo, Japan). The measurement sites were precisely located and marked at the anterior and posterior surface in the middle of the thigh length (the distance from the greater trochanter of the femur to articular cleft between the femur and tibial condyles), and at the anterior and posterior surface in the proximal $30 \%$ of the lower leg length (the distance from the articular cleft to lateral malleolus). The length of the segment was determined using a measuring tape. The position of the subjects during the ultrasonographic measurements, the site selected for obtaining cross-sectional images, and determination of MT at each site were the same as those described in previous studies $[8,9]$. MT at each of the four sites was multiplied by the length of the segment where MT was determined and summed up $\left(\mathrm{MT}_{\mathrm{SUM}} \times \mathrm{L}\right)$.

\section{Dual-energy X-ray absorptiometry measurements}

A whole-body DXA scanner (Delphi A-QDR, Whole body; Hologic, Inc., Bedford, MA, USA) was used to determine fat mass, bone mineral content (BMC) and LTM. The radiation dose was $10 \mu \mathrm{Sv}$. Lower limb image acquisition and analysis were obtained following the manufacturer's instructions. Fat mass, BMC and LTM were calculated using software provided by the manufacturer. A radiation technologist performed DXA measurement and data analysis.

\section{Statistics}

In the model-development group, a multiple regression analysis (stepwise) was applied to a set of independent variables which comprised age, sex, and $\mathrm{MT}_{\mathrm{SUM}} \times \mathrm{L}$ to develop the prediction equation of DXA-based LTM. Sex was coded as a dummy variable: female $=0$ and male $=1$. The difference between the two variables (measured LTM - estimated LTM) was plotted against the mean values of LTM derived from the two methods to examine for systematic error, as described by Bland and Altman [16]. When the equation was validated, it was applied to the cross-validation group. The standard error of the estimate (SEE) was calculated to evaluate the accuracy of the estimate with the equation. The SEE was expressed as both an absolute value and relative to the mean of the measured LTM (\%SEE). Descriptive data are presented as means \pm SDs. A Student's paired t-test was used to test the significance of difference between the measured and estimated LTM. The probability level for statistical significance was set at $P<0.05$. All data analyses were conducted using a statistical software program (SPSS 19.0 for windows, IBM, Japan).

\section{Results}

Descriptive data for the MT and DXA measurements are summarized in Table 1. In the model-development 
Table 1 Physical characteristics of the subjects

\begin{tabular}{|c|c|c|c|c|}
\hline \multirow[t]{2}{*}{ Variables } & \multicolumn{2}{|c|}{ Model-development group $(n=52)$} & \multicolumn{2}{|c|}{ Cross-validation group $(n=25)$} \\
\hline & Men $(n=22)$ & Women $(n=30)$ & Men $(n=11)$ & Women $(n=14)$ \\
\hline Age, years & $62.1 \pm 8.6$ & $66.3 \pm 5.9$ & $67.5 \pm 4.9$ & $63.7 \pm 7.7$ \\
\hline Height, cm & $166.5 \pm 5.6$ & $153.5 \pm 4.1$ & $166.5 \pm 5.6$ & $154.5 \pm 3.0$ \\
\hline Body mass, kg & $65.0 \pm 7.3$ & $50.3 \pm 5.8$ & $67.0 \pm 6.5$ & $53.1 \pm 4.9$ \\
\hline Body mass index, $\mathrm{kg} / \mathrm{m}^{2}$ & $23.4 \pm 2.1$ & $21.4 \pm 2.5$ & $24.2 \pm 2.5$ & $22.2 \pm 1.7$ \\
\hline \multicolumn{5}{|l|}{ Limb length, $\mathrm{cm}$} \\
\hline Thigh & $37.8 \pm 1.9$ & $34.8 \pm 1.5$ & $37.9 \pm 2.0$ & $34.9 \pm 1.5$ \\
\hline Lower leg & $37.0 \pm 2.0$ & $34.2 \pm 1.7$ & $37.1 \pm 2.2$ & $34.1 \pm 1.6$ \\
\hline \multicolumn{5}{|l|}{ MT measurements, $\mathrm{cm}$} \\
\hline Thigh anterior & $4.77 \pm 0.53$ & $3.84 \pm 0.59$ & $4.56 \pm 0.64$ & $3.90 \pm 0.57$ \\
\hline Thigh posterior & $6.12 \pm 0.60$ & $4.85 \pm 0.48$ & $6.29 \pm 0.46$ & $4.98 \pm 0.40$ \\
\hline Lower leg anterior & $3.09 \pm 0.24$ & $2.56 \pm 0.17$ & $3.05 \pm 0.26$ & $2.72 \pm 0.28$ \\
\hline Lower leg posterior & $6.91 \pm 0.38$ & $5.75 \pm 0.37$ & $6.99 \pm 0.61$ & $5.72 \pm 0.39$ \\
\hline \multicolumn{5}{|c|}{ DXA measurements total body } \\
\hline $\mathrm{FM}, \mathrm{kg}$ & $12.1 \pm 3.7$ & $13.8 \pm 3.7$ & $13.1 \pm 2.5$ & $16.4 \pm 2.8$ \\
\hline LTM, kg & $51.5 \pm 4.7$ & $35.7 \pm 3.0$ & $52.4 \pm 4.9$ & $35.6 \pm 2.7$ \\
\hline BMC, kg & $2.2 \pm 0.3$ & $1.5 \pm 0.2$ & $2.2 \pm 0.3$ & $1.6 \pm 0.3$ \\
\hline$\% F M, \%$ & $18.2 \pm 3.9$ & $26.7 \pm 5.0$ & $19.2 \pm 2.8$ & $30.4 \pm 3.3$ \\
\hline \multicolumn{5}{|l|}{ Right leg } \\
\hline $\mathrm{FM}, \mathrm{kg}$ & $1.8 \pm 0.6$ & $2.5 \pm 0.6$ & $2.0 \pm 0.6$ & $3.1 \pm 0.7$ \\
\hline LTM, kg & $8.7 \pm 1.2$ & $5.8 \pm 0.6$ & $8.7 \pm 0.9$ & $6.0 \pm 0.6$ \\
\hline
\end{tabular}

Values are means \pm SDs; thigh length, distance between the lateral condyle of the femur and the greater trochanter; lower leg length, distance between the lateral malleous of the fibula and the lateral condyle of the tibia. BMC, bone mineral content; FM, fat mass; LTM, bone-free lean tissue mass; MT, muscle thickness; $\% \mathrm{FM}$, the percentage of $\mathrm{FM}$ in the total mass.

group, multiple regression analysis produced an equation predicting the measured leg LTM, with only $\mathrm{MT}_{\mathrm{SUM}} \times \mathrm{L}$ as a significant independent variable: leg LTM $(\mathrm{kg})=0.01464 \times$ $\left(\mathrm{MT}_{\mathrm{SUM}} \times \mathrm{L}\right)\left(\mathrm{cm}^{2}\right)-2.767$. The $\mathrm{R}^{2}$ and SEE of this equation were 0.958 and $0.3 \mathrm{~kg}(\% \mathrm{SEE}=4.3 \%)$, respectively (Figure 1$)$. The estimated leg LTM $(7.0 \pm 1.7 \mathrm{~kg})$ did not significantly

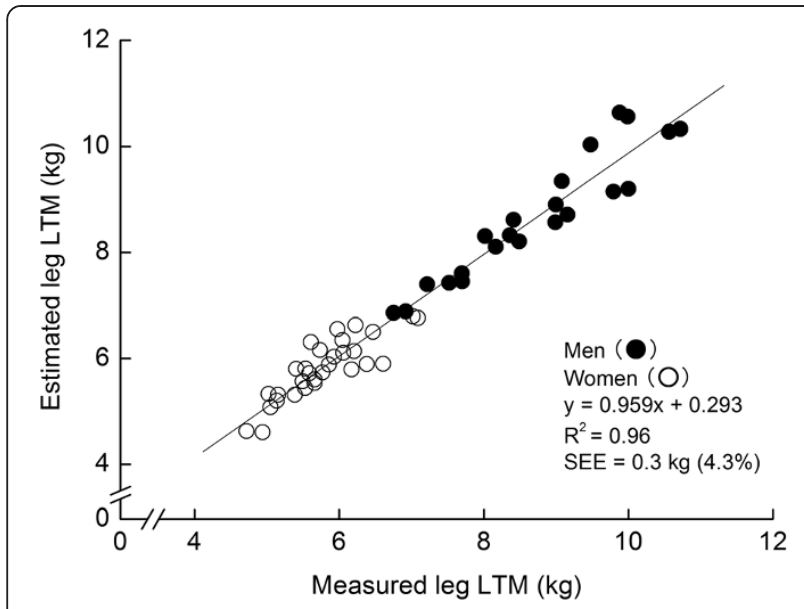

Figure 1 Relationship between the measured and estimated leg lean tissue mass (LTM) in the model-development group. differ from the measured leg LTM $(7.0 \pm 1.7 \mathrm{~kg})$, without a significant systematic error in the Bland-Altman plot (Figure 2). The application of this equation for the cross-validation group did not produce a significant difference between the measured $(7.2 \pm 1.6 \mathrm{~kg})$ or estimated

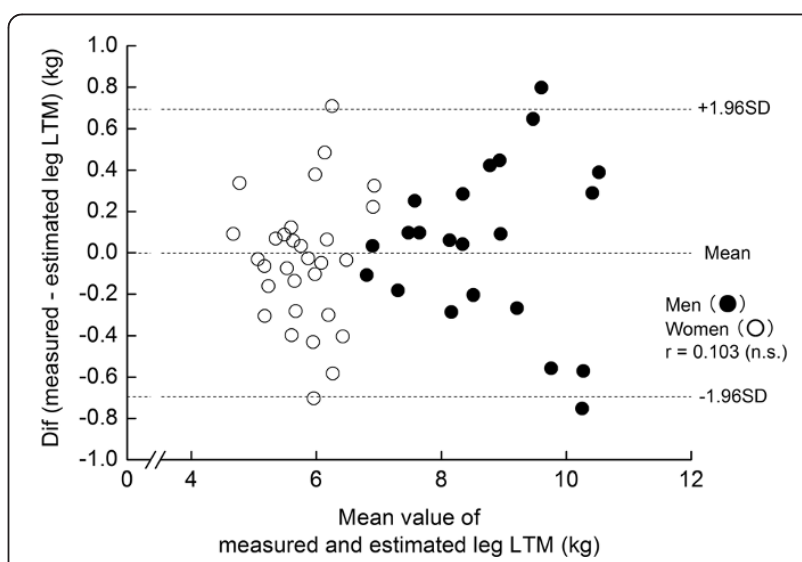

Figure 2 Bland-Altman analysis for the model-development group. Difference between the measured and estimated leg lean tissue mass (LTM) (measured LTM - estimated LTM) versus mean values of the measured and estimated LTM. The dotted horizontal lines are mean differences and 95\% confidence intervals (Cls). 
$(7.2 \pm 1.6 \mathrm{~kg})$ leg LTM and systematic error $(\mathrm{r}=-0.012$, non significant).

\section{Discussion}

The current results support the hypothesis set at the start of this study, and indicate that ultrasound MT measurements have a high potential for estimating leg SM in healthy Japanese middle-aged and older individuals. This study used DXA-based LTM as a variable representing leg SM. It has been shown that DXA-based appendicular SM models overestimate CT-based SM [17,18]. Despite differences in model formulas, however, all are based on the common principle that most leg LTM is SM [14]. Indeed, leg LTM determined by DXA has been shown to be closely correlated to SM measured by CT [13] or MRI [14]. Furthermore, Kim et al. [12] observed a strong association between DXA-based appendicular LTM and MRI-based total SM. These facts and findings indicate that DXA-based LTM is applicable for SM assessment in vivo.

In the present study, we used ultrasonography to determine muscle thickness. The ultrasound-based muscle thickness measurements involve not only muscle but also non-contractile tissues such as intermuscular adipose and connective tissues. Intermuscular adipose tissue has been shown to be increased with aging [19]. Thus, there is a possibility that, for older individuals, the muscle thickness measurements might induce overestimation in lean tissue mass.

Besides ultrasonography, prediction equations with the measures of anthropometry [20-22] or bioelectrical impedance analysis (BIA) [23-26] as independent variables have been developed to estimate lower extremity SM. In these equations, the \%SEE ranged from $3.3 \%$ to $10 \%$. Compared with these values, the \%SEE obtained here, $4.3 \%$, is in the lower rank. Among the previous studies cited above, the lowest \%SEE values reported for each of the anthropometric and BIA approaches are 3.3\% [20] and 3.6\% [26], respectively. These values were obtained from prediction equations derived from anthropometric measurements at multiple levels of limbs [20] or multiple BIA along the limb length [26]. With the ultrasound approach, increasing the number of levels for MT measurements will improve the accuracy of LTM estimation.

\footnotetext{
Abbreviations

B-mode: Brightness-mode; BIA: Bioelectrical impedance analysis; CT: computerized tomography; DXA: dual-energy X-ray absorptiometry; LTM: bone-free lean tissue mass; MRI: magnetic resonance imaging; MT: muscle thickness; SEE: standard error of estimate; SM: skeletal muscle mass; $M T_{\text {SUM }} \times \mathrm{L}$ : sum of products of MT with the length of segment where MT is determined: \%SEE: SEE relative to the mean of the measured LTM.
}

\section{Competing interests}

The authors declare that they have no competing interests.

\section{Authors' contributions}

$Y T$, participated in the study design, coordinated research activities, performed statistical analysis, and drafted the manuscript. MO and RA, participated in the study design. EK and TW, measured and analyzed data of muscle thickness. YK and TF, participated in the study design and coordination, and drafted the manuscript. HK, performed statistical analysis, and drafted the manuscript. All authors read and approved the final manuscript.

\section{Acknowledgement}

This research was supported in part by financial aid from the Ministry of Education, Culture, Sports, Science and Technology of Japan (no. 1248007).

\section{Author details}

${ }^{1}$ National Institute of Fitness and Sports in Kanoya, 1 Shiromizu, Kanoya, Kagoshima 891-2393, Japan. ${ }^{2}$ School of International Liberal studies, Chukyo University, 101 Tokodachi, Kaizu-cho, Toyota-shi, Aichi 470-0393, Japan. ${ }^{3}$ College of Systems Engineering and Science, Shibaura Institute of Technology, 3-7-5 Toyosu, Koto-ku, Tokyo 135-8548, Japan. ${ }^{4}$ Department of Sports Sciences, Japan Institute of Sports Sciences, 3-15-1 Nishigaoka Kita-ku, Tokyo 115-0056, Japan. ${ }^{5}$ Faculty of Health and Sports Science, Doshisha University, 1-3 Tatara Miyakodani, Kyotanabe-shi, Kyoto-fu 610-0394, Japan.

${ }^{6}$ Faculty of Sports Science, Waseda university, 2-579-15 Mikajima,

Tokorozawa, Saitama 359-1192, Japan.

Received: 8 May 2013 Accepted: 14 September 2013 Published: 25 September 2013

\section{References}

1. Janssen I, Heymsfield SB, Wang ZM, Ross R: Skeletal muscle mass and distribution in 468 men and women aged 18-88 yr. J Appl Physiol 2000, 89:81-88.

2. McDonagh MJ, White MJ, Davies CT: Different effects of ageing on the mechanical properties of human arm and leg muscles. Gerontology 1984 30:49-54.

3. Lynch NA, Metter EJ, Lindle RS, Fozard JL, Tobin JD, Roy TA, Fleg JL, Hurley BF: Muscle quality. I. Age-associated differences between arm and leg muscle groups. J Appl Physiol 1999, 86:188-194

4. Runnels ED, Bemben DA, Anderson MA, Bemben MG: Influence of age on isometric, isotonic, and isokinetic force production characteristics in men. J Geriatr Phys Ther 2005, 28:74-84.

5. Hortobagyi T, Mizelle C, Beam S, DeVita P: Old adults perform activities of daily living near their maximal capabilities. J Gerontol A Biol Sci Med Sci 2003, 58:M453-M460.

6. Nakamoto H, Yoshitake Y, Takai Y, Kanehisa H, Kitamura T, Kawanishi M, Mori S: Knee extensor strength is associated with Mini-Mental State Examination scores in elderly men. Eur J Appl Physiol 2012, 112:1945-1953.

7. Nourhashemi F, Andrieu S, Gillette-Guyonnet S, Reynish E, Albarede JL, Grandjean $\mathrm{H}$, Vellas B: Is there a relationship between fat-free soft tissue mass and low cognitive function? Results from a study of 7,105 women. J Am Geriatr Soc 2002, 50:1796-1801.

8. Abe T, Sakamaki M, Yasuda T, Bemben MG, Kondo M, Kawakami Y Fukunaga T: Age-related, site-specific muscle loss in 1507 Japanese men and women aged 20 to 95 years. J Sports Sci Med 2011, 10:145-150.

9. Abe T, Kondo M, Kawakami Y, Fukunaga T: Prediction equations for body composition of Japanese adults by B-mode ultrasound. Am J Hum Biol 1994, 6:161-170

10. Miyatani M, Kanehisa H, Kuno S, Nishijima T, Fukunaga T: Validity of ultrasonograph muscle thickness measurements for estimating muscle volume of knee extensors in humans. Eur J Appl Physiol 2002, 86:203-208.

11. Miyatani M, Kanehisa H, Ito M, Kawakami Y, Fukunaga T: The accuracy of volume estimates using ultrasound muscle thickness measurements in different muscle groups. Eur J Appl Physiol 2004, 91:264-272.

12. Kim J, Wang Z, Heymsfield SB, Baumgartner RN, Gallagher D: Total-body skeletal muscle mass: estimation by a new dual-energy X-ray absorptiometry method. Am J Clin Nutr 2002, 76:378-383.

13. Levine JA, Abboud L, Barry M, Reed JE, Sheedy PF, Jensen MD: Measuring leg muscle and fat mass in humans: comparison of $C T$ and dual-energy X-ray absorptiometry. J App/ Physio/ 2000, 88:452-456.

14. Shih R, Wang Z, Heo M, Wang W, Heymsfield SB: Lower limb skeletal muscle mass: development of dual-energy X-ray absorptiometry prediction model. J App/ Physiol 2000, 89:1380-1386. 
15. Dupler TL, Tolson $\mathrm{H}$ : Body composition prediction equations for elderly men. J Gerontol A Biol Sci Med Sci 2000, 55:M180-M184.

16. Bland JM, Altman DG: Statistical methods for assessing agreement between two methods of clinical measurement. Lancet 1986, 1:307-310.

17. Wang W, Wang Z, Faith MS, Kotler D, Shih R, Heymsfield SB: Regional skeletal muscle measurement: evaluation of new dual-energy X-ray absorptiometry model. J Appl Physiol 1999, 87:1163-1171.

18. Visser M, Fuerst T, Lang T, Salamone L, Harris TB: Validity of fan-beam dual-energy X-ray absorptiometry for measuring fat-free mass and leg muscle mass. Health, Aging, and Body Composition Study-Dual-Energy X-ray Absorptiometry and Body Composition Working Group. J Appl Physiol 1999, 87:1513-1520.

19. Buford TW, Lott DJ, Marzetti E, Wohlgemuth SE, Vandenborne K, Pahor M, Leeuwenburgh C, Manini TM: Age-related differences in lower extremity tissue compartments and associations with physical function in older adults. Exp Gerontol 2012, 47:38-44.

20. Tothill P, Stewart AD: Estimation of thigh muscle and adipose tissue volume using magnetic resonance imaging and anthropometry. J Sports Sci 2002, 20:563-576.

21. Chen BB, Shih TTF, Hsu CY, Yu CW, Wei SY, Chen CY, Wu CH, Chen CY: Thigh muscle volume predicted by anthropometric measurements and correlated with physical function in the older adults. J Nutr Health Aging 2011, 15:433-438.

22. Wen $X$, Wang $M$, Jiang CM, Zhang YM: Anthropometric equation for estimation of appendicular skeletal muscle mass in Chinese adults. Asia Pac J Clin Nutr 2011, 20:551-556.

23. Fuller NJ, Hardingham CR, Graves M, Screaton N, Dixon AK, Ward LC, Elia M: Predicting composition of leg sections with anthropometry and bioelectrical impedance analysis, using magnetic resonance imaging as reference. Clin Sci 1999, 96:647-657.

24. Miyatani M, Kanehisa H, Masuo Y, Ito M, Fukunaga T: Validity of estimating limb muscle volume by bioelectrical impedance. J Appl Physiol 2001, 91:386-394.

25. Kyle UG, Genton L, Hans D, Pichard C: Validation of a bioelectrical impedance analysis equation to predict appendicular skeletal muscle mass (ASMM). Clin Nutr 2003, 22:537-543.

26. Stahn A, Terblanche E, Strobel G: Modeling upper and lower limb muscle volume by bioelectrical impedance analysis. J Appl Physiol 2007 103:1428-1435.

doi:10.1186/1880-6805-32-12

Cite this article as: Takai et al.: Validity of ultrasound muscle thickness measurements for predicting leg skeletal muscle mass in healthy Japanese middle-aged and older individuals. Journal of Physiological Anthropology 2013 32:12.

\section{Submit your next manuscript to BioMed Central and take full advantage of:}

- Convenient online submission

- Thorough peer review

- No space constraints or color figure charges

- Immediate publication on acceptance

- Inclusion in PubMed, CAS, Scopus and Google Scholar

- Research which is freely available for redistribution 